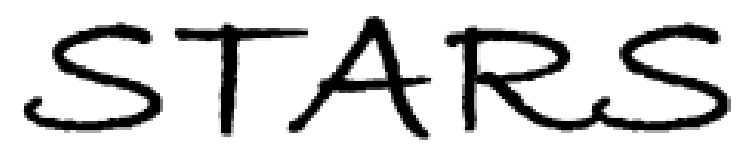

University of Central Florida

STARS

$1-1-2003$

\title{
Second harmonic generation tuning curves in quasiphase- matched potassium titanyl phosphate with narrow, high-intensity beams
}

Mordechai Katz

David Eger

Hongki Kim

University of Central Florida

Ladislav Jankovic

University of Central Florida

George Stegeman

University of Central Florida

Find similar works at: https://stars.library.ucf.edu/facultybib2000

University of Central Florida Libraries http://library.ucf.edu See next page for additional authors

This Article is brought to you for free and open access by the Faculty Bibliography at STARS. It has been accepted for inclusion in Faculty Bibliography 2000s by an authorized administrator of STARS. For more information, please contactSTARS@ucf.edu.

\section{Recommended Citation}

Katz, Mordechai; Eger, David; Kim, Hongki; Jankovic, Ladislav; Stegeman, George; Carrasco, Silvia; and Torner, Lluis, "Second harmonic generation tuning curves in quasiphase-matched potassium titanyl phosphate with narrow, high-intensity beams" (2003). Faculty Bibliography 2000s. 3849.

https://stars.library.ucf.edu/facultybib2000/3849

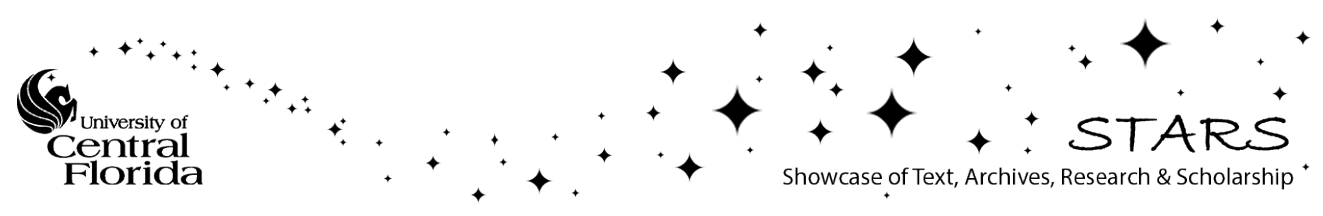




\section{Authors}

Mordechai Katz, David Eger, Hongki Kim, Ladislav Jankovic, George Stegeman, Silvia Carrasco, and Lluis Torner 


\section{Second harmonic generation tuning curves in quasiphase-matched potassium titanyl phosphate with narrow, high-intensity beams}

Cite as: Journal of Applied Physics 93, 8852 (2003); https://doi.org/10.1063/1.1563302 Submitted: 31 July 2002 . Accepted: 03 February 2003. Published Online: 19 May 2003

Mordechai Katz, David Eger, Hongki Kim, Ladislav Jankovic, George Stegeman, Silvia Carrasco, and Lluis Torner

\section{ARTICLES YOU MAY BE INTERESTED IN}

Continuous-wave self-pumped optical parametric oscillator based on $\mathrm{Yb}^{3+}$-doped bulk periodically poled $\mathrm{LiNbO}_{3}(\mathrm{MgO})$

Applied Physics Letters 79, 293 (2001); https://doi.org/10.1063/1.1383567

Parametric Interaction of Focused Gaussian Light Beams

Journal of Applied Physics 39, 3597 (1968); https://doi.org/10.1063/1.1656831

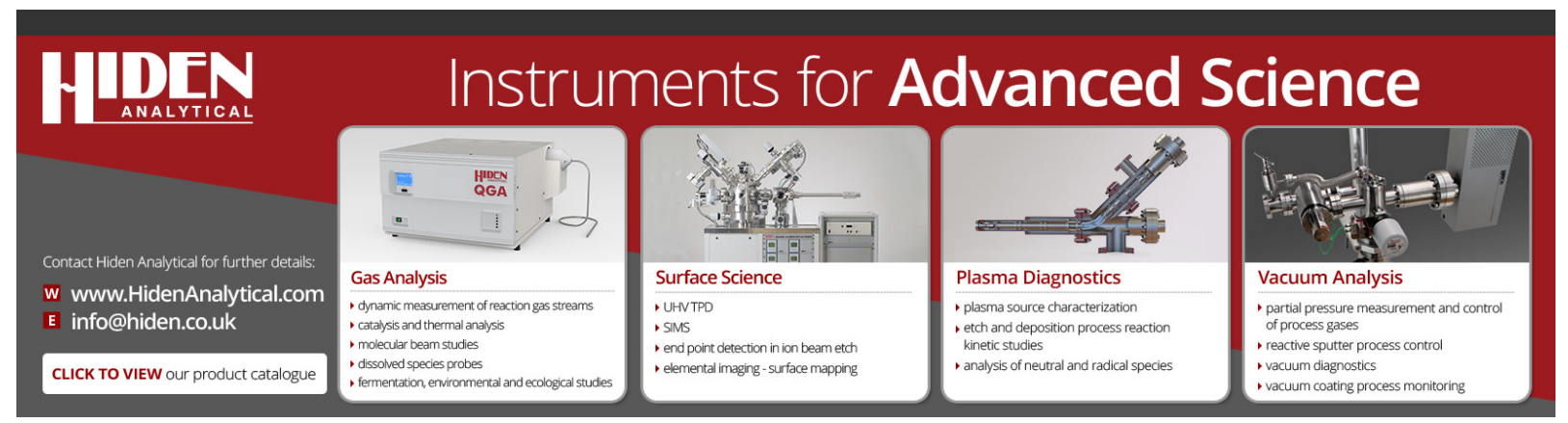




\title{
Second harmonic generation tuning curves in quasiphase-matched potassium titanyl phosphate with narrow, high-intensity beams
}

\author{
Mordechai Katz ${ }^{\text {a) }}$ and David Eger \\ Electro-Optic Division, Soreq NRC, Yavene, 81800, Israel \\ Hongki Kim, Ladislav Jankovic, and George Stegeman \\ CREOL/School of Optics, University of Central Florida, Orlando, Florida 32816-2700 \\ Silvia Carrasco and Lluis Torner \\ Institute of Photonic Sciences, and Department of Signal Theory and Communications, \\ Universitat Politecnica de Catalunya, 08034 Barcelona, Spain
}

(Received 31 July 2002; accepted 3 February 2003)

\begin{abstract}
The second harmonic generation (SHG) tuning curves with temperature and angle of incidence were measured in periodically poled $\mathrm{KTiOPO}_{4}$ for narrow fundamental beams at intensities typical of quadratic soliton generation. Mutual self-focusing of the fundamental and harmonic, cascading, and walk-off for light incidence away from the poling axis drastically distorted the SHG response curves, in good agreement with theory. (C) 2003 American Institute of Physics.
\end{abstract}

[DOI: $10.1063 / 1.1563302]$

\section{INTRODUCTION}

Second harmonic $(\mathrm{SH})$ generation $(\mathrm{SHG})$ in particular, and parametric mixing in general have been very valuable and versatile techniques for extending the frequency range of lasers since the early days of nonlinear optics. ${ }^{1}$ Until the advent of quasiphase-matching (QPM) in the late 1980s, the phase-matching techniques used for efficient frequency conversion were either Type I (one fundamental and one harmonic wave) or Type II (two fundamental and one harmonic waves) in which both orthogonal polarizations participate. ${ }^{2,3}$ For plane-wave (very wide) input beams there is essentially a single input wave vector ( $\delta$-function angular distribution). In this limit and for low-conversion efficiency, the relative SHG response is given by $\operatorname{sinc}^{2}(\Delta \varphi / 2)$ where $\Delta \varphi$ is the "phasemismatch." The simplest expression for $\Delta \varphi$ occurs for Type I and is given by $\Delta \varphi=\left[2 k_{1}-k_{2}\right] L=2 \omega\left[n_{1}-n_{2}\right] L / c$ where $L$ is the sample length, $\omega$ is the fundamental frequency, $k_{1}$ and $k_{2}$ are the fundamental (FW) and harmonic wave vectors, and the $n_{1}$ and $n_{2}$ are the corresponding refractive indices. That is, phase-matching occurs when the index ellipsoids for the $\mathrm{FW}\left(n_{1}\right)$ and $\mathrm{SH}\left(n_{2}\right)$ intersect (called "critical phase-matching," CPM), or just touch ("noncritical phasematching," NCPM). The sinc ${ }^{2}$ response is distorted for nonnegligible harmonic generation with the width of the principal maximum narrowing and the sidebands increasing in intensity and collapsing towards $\Delta \varphi=0 .{ }^{4}$ Experimental measurements over the years of the SHG efficiency versus phase mismatch for relatively wide beams have confirmed these well-known forms for the tuning curves. ${ }^{5,6}$

Narrow (finite width) beams have a distribution of incident wave vectors and the local intensity varies with position in the sample. This distorts and broadens the $\operatorname{sinc}^{2}$ response. The optimization of the SHG (or parametric mixing) re-

\footnotetext{
a)Electronic mail: moti@soreq.gov.il
}

sponse by focusing the incident beam roughly in the center of the sample and by choosing the optimum minimum spot size has been well-understood for a long time and is the basis for commercial doubling crystals. ${ }^{7-9}$

The development of QPM has provided a powerful technique for producing phase matching for parametric processes like second harmonic generation at virtually any wavelength just by changing the period of the poling. ${ }^{10,11}$ Furthermore, this process usually allows the large diagonal elements of the nonlinearity tensor in noncentrosymmetric ferroelectric media to be used with propagation along a crystal symmetry axis. This typically results in noncritical phase-matching (NCPM) with all its attractive features. Copolarized FW and SH beams are used and the phase mismatch for SHG generated along the appropriate crystal axis. The phase mismatch now takes the form $\Delta \varphi=\left[2 k_{1}-k_{2}+2 \pi m / \Lambda\right] L=2 \omega\left[n_{1}\right.$ $\left.-n_{2}+\pi m c / \Lambda \omega\right] L / c$ where $\Lambda$ is the period of the poling and $m$ is the $m$ th harmonic grating wave vector. ${ }^{11}$ Note that off-axis propagation leads to "walk-off" of the FW from the SH that occurs at an angle $\psi$ given by $\sin \psi=\sin \theta \kappa / k_{2}(\kappa$ $=2 \pi / \Lambda$ ) for internal incidence angle $\theta$ from the $\mathrm{X}$ axis. ${ }^{11}$ The distortion in the SHG tuning curves near phase match for narrow beams has been confirmed experimentally in QPM samples, for example, in periodically poled $\mathrm{KTiOPO}_{4}$ (PPKTP) which is of principal interest here. ${ }^{12-14}$ With narrow incident beams, the SHG tuning response for QPM systems is very similar, but not identical to that discussed above for Type I, NCPM, due to the induced walk-off that appears in the case of propagation away from the NCPM direction. The effect of QPM near the poling axis can be approximately illustrated by adding its contribution to the FW index ellipsoid to distort it as shown in Fig. 1.

However, it is known that narrow beams, but with much higher intensities than typically used for SHG, are used to generate spatial solitons in quadratically nonlinear media. ${ }^{15-20}$ Usually the incident FW is focused onto the in- 

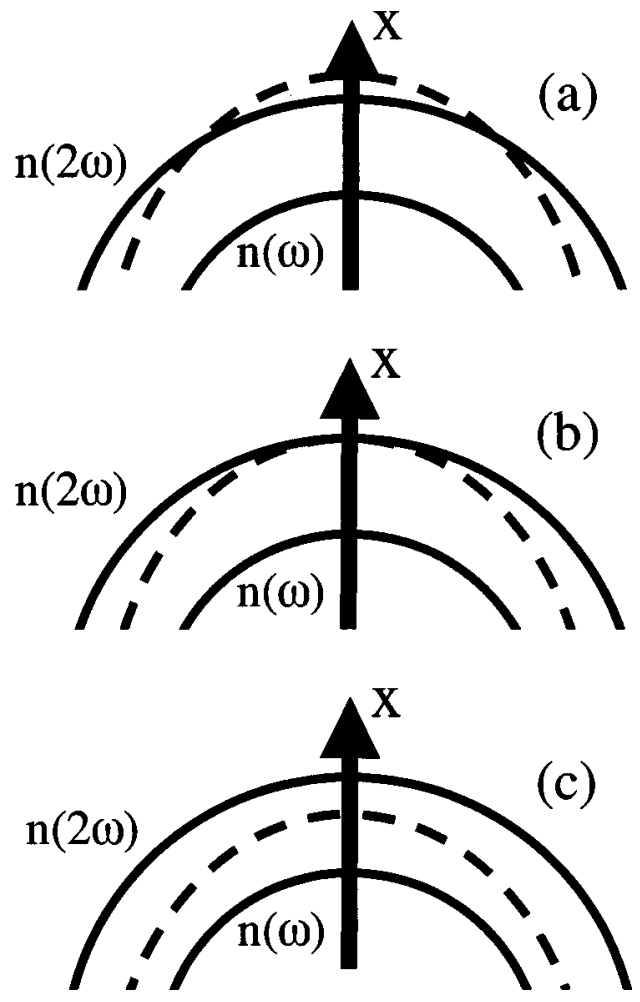

FIG. 1. Cuts of the index ellipsoids in the X-Y plane for the Z-polarized FW and $\mathrm{SH}$. The dashed line is meant to simulate approximately the effect of the nonlinear QPM grating on phase-matching. (a) is for temperatures below $T_{\mathrm{PM}}$, (b) is at the phase-matching temperature, and (c) $T>T_{\mathrm{PM}}$.

put surface of a doubling crystal with parameters near a phase-matching condition. It is well-known in nonlinear optics from the structure of the nonlinear polarization that wave mixing always leads to a nonlinearly generated beam that is narrower in space than the input beams being mixed. This beam narrowing also occurs for both the $\mathrm{SH}$ and $\mathrm{FW}$ via

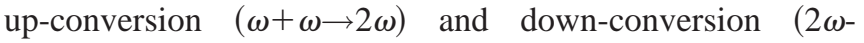
$\omega \longrightarrow \omega)$, respectively, leading to a progressive narrowing of both beams. $^{21,22}$ In addition, cascading which produces nonlinear phase shifts due to the differential phase velocity of the waves away from phase matching also contributes to beam focusing for $\Delta k>0$, and to defocusing for $\Delta k<0 .{ }^{22,23}$ Nevertheless, it is important to stress that finite beams do not carry a single wave vector, but rather a set of wave vectors given by the corresponding Fourier plane-wave expansion. Therefore, for a finite beam the nominal value of $\Delta k$ measures the wave-vector mismatch along only one of the wave vectors present. Therefore, when $\Delta k=0$ the "additional" wave vectors are not phase matched, and thus cascading does occur at $\Delta k=0$, too. Notice also that for high-input peak powers, the hypothesis of very low SH required does not hold, and thus the simple concept of self-focusing and selfdefocusing mentioned above must be taken with caution. In particular, self-focusing does occur at $\Delta k<0$ under appropriate conditions, typically associated with a large $\mathrm{SH}$.

When beam narrowing processes are balanced by the diffraction of both beams, a quadratic soliton is generated. For beams with $20 \mu \mathrm{m}$ waists, and nonlinearities of order 10 $\mathrm{pm} / \mathrm{V}$, intensities of a few $\mathrm{GW} / \mathrm{cm}^{2}$ are typically required to generate solitons. These values are in a different (orders of magnitude higher) regime than those used previously for SHG and hence the SHG tuning response can be quite different. In fact the length scaling parameters important in this limit differ from those for the usual SHG. For example, the parametric gain length replaces the actual sample length as the parameter that determines bandwidth, etc. Furthermore, in CPM geometries, walk-off between the fundamental and harmonic beams frequently occurs, further adding to the distortion that a beam can experience. Thus nonlinear interaction effects involving the second-order nonlinearity such as nonlinear beam narrowing, cascading, soliton generation, and walk-off can all play key roles, sometimes competing, in changing the shape of the interacting beams. In turn, they affect the net SHG.

Quadratic spatial solitons have already been observed in the periodically poled doubling crystal $\mathrm{LiNbO}_{3}$, namely, PPLN, in which the lowest measured soliton threshold of 1.5 $\mathrm{GW} / \mathrm{cm}^{2}$ was reported. ${ }^{19}$ However, the question of the SHG tuning from phase match in the intensity regime associated with soliton generation has only been addressed experimentally by Schiek and coworkers in the specific case of titanium-indiffused lithium niobate optical channel waveguides with nonuniform wave-vector mismatches along the waveguide. ${ }^{24}$ In fact SHG with input intensities of $10 \mathrm{~s}$ of $\mathrm{GW} / \mathrm{cm}^{2}$ have been used to study periodically poled KTP, PPKTP, but no SHG tuning curves, or soliton generation were reported. ${ }^{12}$ In this paper, we investigate experimentally the SHG response curves for periodically poled KTP at multi-GW/cm ${ }^{2}$ input intensities. We show large asymmetries in the tuning curves with temperature (equivalent to wavelength) and incidence angle for narrow input beams and identify several features in both the output beam profiles and tuning curves. Measured beam shapes at the output were found to be very useful in interpreting the mechanisms that are active. All of the experimental results are in good agreement with theoretical trends based on the usual coupled mode equations.

\section{EXPERIMENTAL DETAILS}

KTP is a biaxial ferroelectric crystal which can be made NCPM via QPM for propagation along the crystal's X axis. The samples were fabricated at Soreq (Israel) using the lowtemperature poling technique. ${ }^{25,26}$ Periodic poling to reverse the direction of the nonlinearity along the $\mathrm{Z}$ axis utilizes the $d_{33}$ coefficient reported to be $16.9 \mathrm{pm} / \mathrm{V} \pm 10 \%$ in bulk crystals. ${ }^{27}$ Various implementations of the Soreq bulk PPKTP samples to SHG yielded effective QPM values of $d_{\text {eff }}$ $\left(=2 d_{33} / \pi\right)$ of $9.5 \mathrm{pm} / \mathrm{V}$ which corresponds to a $d_{33}$ $=14.9 \mathrm{pm} / \mathrm{V} \cdot{ }^{28-31}$ For a poling period of $8.99 \mu \mathrm{m}$, phase matching is calculated for a $1064 \mathrm{~nm}$ fundamental beam input to occur around $40{ }^{\circ} \mathrm{C}$ from the published data on refractive index..$^{31,32}$ The sample was $1 \mathrm{~cm}$ long and the width and thickness of the PPKTP were 4.5 and $0.5 \mathrm{~mm}$ (along Z), respectively. The measured $\mathrm{SHG}$ bandwidth was $0.2 \mathrm{~nm}$, in good agreement with theory and an indication that phase matching is realized over the full length of the crystal. ${ }^{29}$ In SHG experiments on similar samples, maximum intensities 


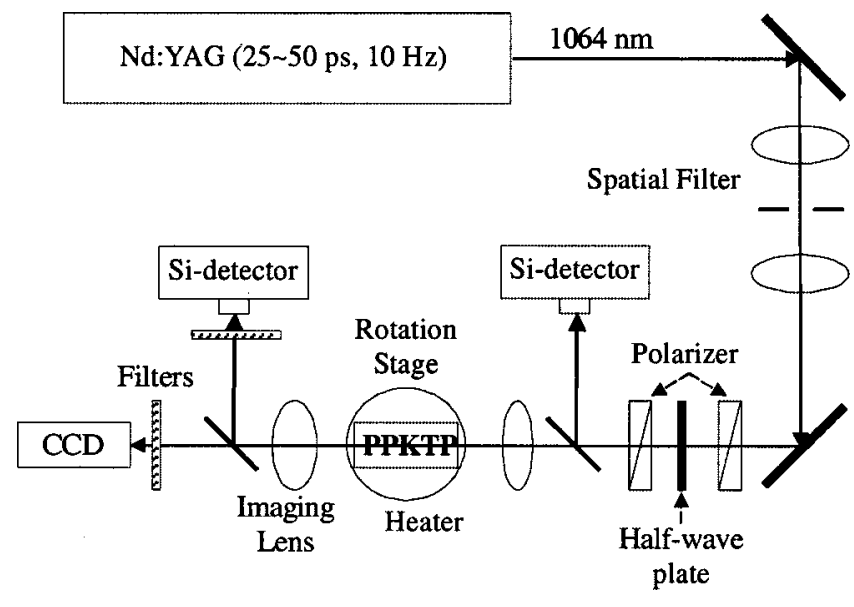

FIG. 2. Measurement setup.

have been as high as a few $\mathrm{MW} / \mathrm{cm}^{2}$ and have produced large conversion efficiencies (up to $60 \%$ ) for $280 \mu \mathrm{m}$ beam waist diameters $\left(1 / e^{2}\right)$ at the crystal. ${ }^{29}$

The experimental system used is shown schematically in Fig. 2. The laser source was an EKSMA Nd:YAG laseramplifier combination which produced $100 \mathrm{~mJ}$ pulses (of which a few $\mu \mathrm{J}$ were used in the experiments), duration 25 or $50 \mathrm{ps}$ (selectable) at $10 \mathrm{~Hz}$. The $10 \mathrm{~mm}$ diameter amplifier rod operates in the "under-filled" regime and ensures a truly quasi-Gaussian beam spatial profile rather than the "flat top" typical of overfilled rods. As a result the output beam from the laser was highly cylindrically symmetric, and was improved even further by spatial filtering. The measured $\mathbf{M}^{2}$ was 1.1-1.2. The full width at half maximum spectral bandwidth was measured to vary from 0.11 to $0.14 \mathrm{~nm}$, about a factor of two smaller than the SHG bandwidth.

The $\mathrm{Z}$ polarized $1064 \mathrm{~nm}$ beam was focused by lenses to minimum beam waists $w_{0}=40$ (for approximating the plane wave limit) and $\sim 16 \mu \mathrm{m}$ (for the narrow beam experiments), as measured by the knife-edge method. Most of the experiments were done with a minimum waist of $16.4 \mu \mathrm{m}$ which gives 7.5 diffraction lengths of propagation along the $\mathrm{X}$ axis. Normally the crystal's input facet was placed at the position of the minimum beam waist, the usual geometry for exciting spatial solitons. The largest intensity input into the crystal was $\sim 50 \mathrm{GW} / \mathrm{cm}^{2}$ with no visible damage observed over either the short or long terms. The individual beams emerging from the back face of the crystal were directed onto a camera and a detector to measure their pulse energy and spatial distribution.

\section{THEORETICAL BACKGROUND}

In the absence of diffraction, i.e., for continuouswave plane waves, the coupled mode equations which describe the interaction between copolarized fundamental $\left(\mathrm{FW} \propto a_{1} \exp \left[i\left(\omega t-k_{1} z\right)\right]\right)$ and second harmonic $\left(\mathrm{SH} \propto a_{2} \exp \left[i\left(2 \omega t-k_{2} z\right)\right]\right)$ fields under conditions of noncritical phase matching are well-known: ${ }^{32}$

$$
\frac{d a_{1}}{d z}+i \sqrt{\eta_{0}} a_{1}^{*} a_{2} \exp [-i \Delta k z]=0,
$$

$$
\frac{d a_{2}}{d z}+i \sqrt{\eta_{0}} a_{1}^{2} \exp [i \Delta k z]=0,
$$

with solutions given by, 5,33

$$
\eta=\frac{P_{2}(L)}{P_{1}(0)}=v_{b}^{2} s n^{2}\left(\frac{\Gamma L}{v_{b}}, v_{b}^{4}\right),
$$

with

$$
v_{b}=\frac{1}{\Delta k / 4 \Gamma}+\sqrt{1+(\Delta k / 4 \Gamma)^{2}},
$$

$\Delta k=2 k_{1}-k_{2}$ is the wave-vector mismatch, $\ell_{p g}=\Gamma^{-1}$ $=\sqrt{\eta_{0} P_{1}(0)}$ is the normalized parametric gain length, $\eta_{0}$ is the normalized conversion efficiency (in units of $\mathrm{W}^{-1} \mathrm{~cm}^{-2}$ ), $L$ is the crystal length, and $s n$ is a Jacobi elliptic function. In the limit of weak SHG this expression simplifies to the wellknown $\eta=P_{1}(0) \eta_{0} L^{2} \sin c^{2}(\Delta k L / 2)$.

When augmented to include diffraction and the periodic sign reversal of the quadratic nonlinearity induced by the periodical poling, the equations can be written in the form ${ }^{34}$

$$
\begin{aligned}
& i \frac{\partial a_{1}}{\partial z}-\frac{1}{2 k_{1}}\left\{\frac{\partial^{2}}{\partial y^{2}}+\frac{\partial^{2}}{\partial x^{2}}\right\} a_{1}+\Gamma a_{1}^{*} a_{2} \exp [-i \Delta k z]=0, \\
& i \frac{\partial a_{2}}{\partial z}-\frac{1}{2 k_{2}}\left\{\frac{\partial^{2}}{\partial y^{2}}+\frac{\partial^{2}}{\partial x^{2}}\right\} a_{2}+\Gamma a_{1}^{2} \exp [i \Delta k z]=0,
\end{aligned}
$$

where $\Delta k=2 k_{1}-k_{2}, \Gamma$ changes sign periodically along the poling axis with period $\Lambda,|\Gamma| \propto d_{\text {eff }}^{(2)}$, and $d_{\mathrm{eff}}^{(2)}=2 d_{33}^{(2)} / \pi$. For propagation on-axis (i.e., temperature tuning), the nonlinearity varies periodically along the propagation direction, hence $\Gamma=\Gamma(z)$; for propagation off-axis (i.e., angle tuning) the nonlinearity varies also along the transverse coordinate, thus $\Gamma=\Gamma(x, z)$. For propagation along the poling axis, if the contribution of the QPM is added to the fundamental's refractive index surface, the cuts of the index surfaces which correspond to phase-matching in the crystal's X-Y plane are shown in Fig. 1. These equations describe the universal interaction between the FW and the $\mathrm{SH}$ in the absence of loss. Included are the effects of mutual beam narrowing due to the exchange of photons between the two beams and cascading. Beam narrowing occurs for all nonlinear wave mixing processes and has a simple origin in the SHG case. Assume that the fundamental field is a Gaussian of the form $\exp \left[-r^{2} / w_{0}^{2}\right]$. Since the harmonic that is generated is proportional to $a_{1}^{2}$, its spatial width is proportional to $\exp \left[-2 r^{2} / w_{0}^{2}\right]$, i.e., its width is $w_{0} / \sqrt{2}$, narrower than the fundamental. Similarly, down-conversion $(2 \omega-\omega \longrightarrow \omega)$ involves the generation of the fundamental via $a_{2} a_{1}^{*} \propto \exp \left[-3 r^{2} / w_{0}^{2}\right]$, i.e., also leads to beam narrowing. Therefore both the fundamental and harmonic are narrowed. In addition, changes in the beam profile due to cascading have a similarly simple origin. For $\Delta k \neq 0$, the two waves, harmonic and fundamental, travel at different phase velocities. Thus the fundamental field returning to the fundamental from the harmonic via down-conversion is not in phase with the unconverted fundamental and hence either retards or advances its phase. Far off phase matching, this induces an 


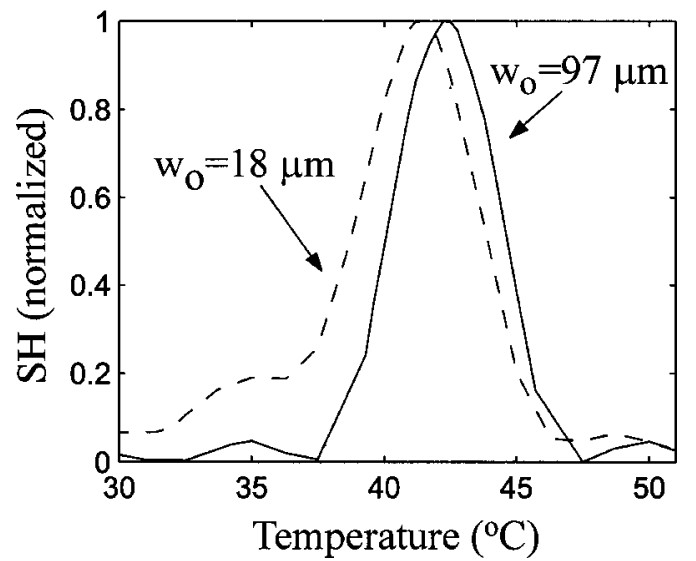

FIG. 3. Effect of decreasing the input beam width on SHG tuning curves at low powers. Solid lines: $\mathrm{w}_{0}=97 \mu \mathrm{m}$ and input power of $0.04 \mathrm{~W}(270$ $\left.\mathrm{W} / \mathrm{cm}^{2}\right)$; dashed lines: $\mathrm{w}_{0}=18 \mu \mathrm{m}$ and $1.12 \mathrm{~W}\left(0.22 \mathrm{MW} / \mathrm{cm}^{2}\right)$. To avoid confusion between experimental data taken with pulsed light and $\mathrm{cw}$ numerical simulations, throughout the paper the former are given in terms of peak intensity and the latter are always given in power.

effective $\chi^{(3)}$ which in the limit of plane waves can be expressed as an effective nonlinear refractive index coefficient $n_{2}\left(\Delta n=n_{2} I\right)$ as ${ }^{35,36}$

$$
n_{2, \mathrm{eff}}=\frac{\pi\left[\chi_{\mathrm{eff}}^{(2)}\right]^{2}}{2 \epsilon_{0} c n^{4} \lambda \Delta k}[1-\cos (\Delta k z)],
$$

so that at this low-depletion, plane-wave limit self-focusing of the fundamental occurs for $\Delta k>0$ and self-defocusing for $\Delta k<0$. As mentioned above, it is worth stressing here that
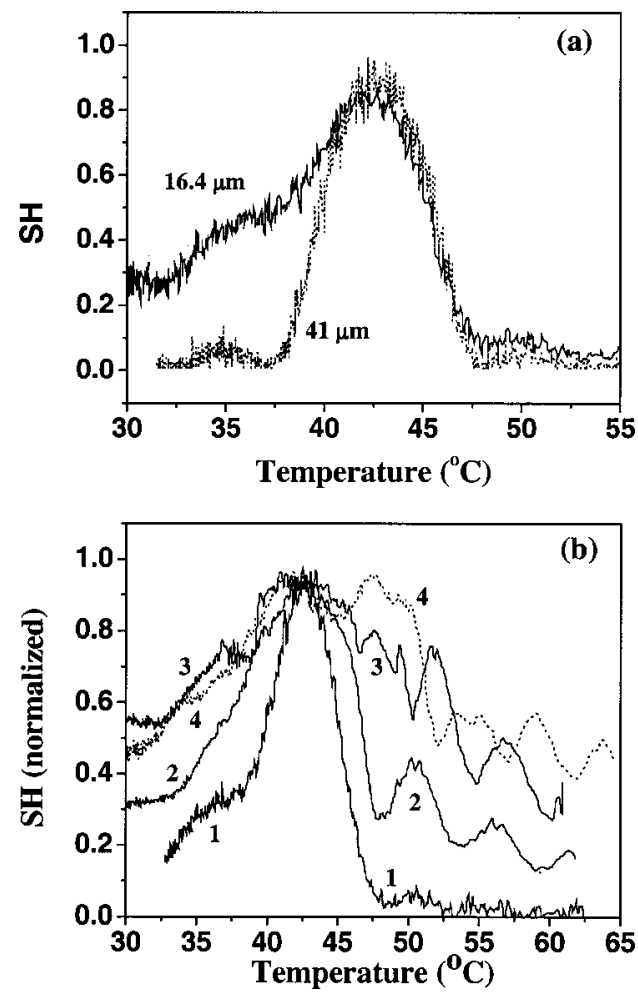

FIG. 4. Tuning curves for SHG obtained by temperature tuning around the low-intensity phase-match temperature $\left(42.7^{\circ} \mathrm{C}\right)$. (a) Input intensity of 11 $\mathrm{KW} / \mathrm{cm}^{2}$ for two different input beam widths. (b) 1-0.02, 2-2.0, 3-6.1, and $4-12.0 \mathrm{GW} / \mathrm{cm}^{2}$.
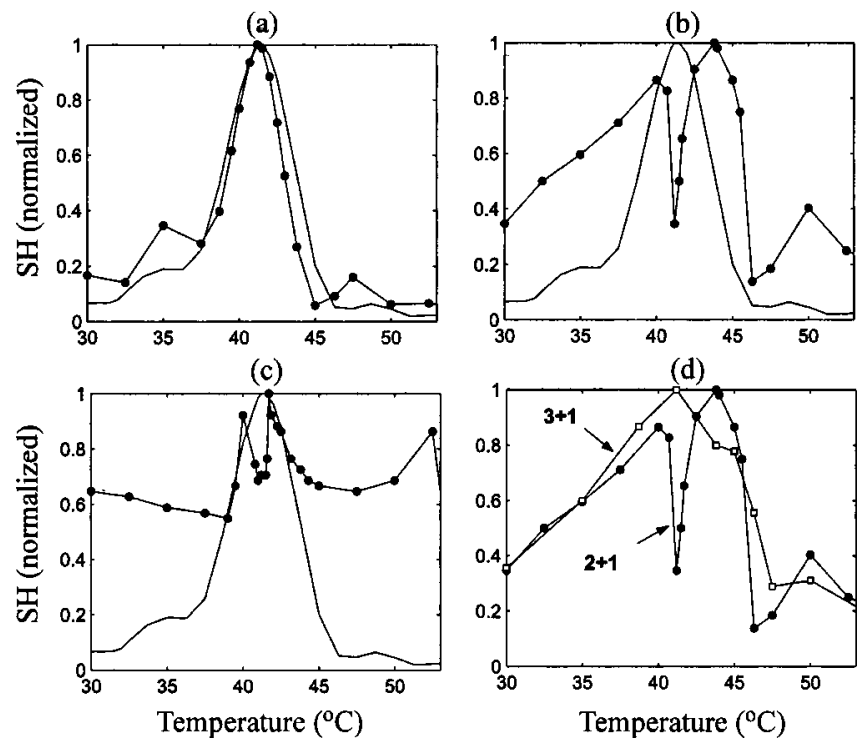

FIG. 5. Calculated continuous-wave SHG tuning curves for temperature detuning from phase-match, with and input beam of $18 \mu \mathrm{m}$, and a crystal length of $10 \mathrm{~mm}$ for different input intensities. Solid lines: cw simulations for $1.12 \mathrm{~W}\left(0.22 \mathrm{MW} / \mathrm{cm}^{2}\right)$. Filled circles: $\mathrm{cW}$ simulations for input powers of (a) $0.45 \mathrm{KW}\left(88 \mathrm{MW} / \mathrm{cm}^{2}\right)$, (b) $2.8 \mathrm{KW}\left(550 \mathrm{MW} / \mathrm{cm}^{2}\right)$, and (c) $13.5 \mathrm{KW}$ $\left(2.6 \mathrm{GW} / \mathrm{cm}^{2}\right)$. In (d), the results of $(3+1)$ spatiotemporal simulations for 20 ps pulses are compared with the $\mathrm{cw}(2+1)$ case for the input power $2.8 \mathrm{KW}$ $\left(550 \mathrm{MW} / \mathrm{cm}^{2}\right)$.

this simple picture has to be used with caution with focused beams and in the presence of an intense SH signal, when, e.g., self-focusing does occur at a negative wave-vector mismatch. $^{37-43}$

The above equations can be used for calculating and optimizing the SHG efficiency for beams of arbitrary width, or the properties of quadratic solitons. However the fields exhibit different properties in the low-depletion and soliton limits. ${ }^{7,8,37-44}$ For efficient SHG on phase match the two fields are $\pi / 2$ out of phase and energy flows from one field into the other with distance. ${ }^{2,3}$ Off phase match, the relative phase between the fields rotates with distance and there is a periodic energy exchange between the fields. For quadratic solitons, the amplitudes and relative phases of the two field components, $a_{1}(\mathrm{FW})$ and $a_{2}(\mathrm{SH})$, are both constant with distance, irrespective of the initial wave-vector mismatch (which is defined in the linear or low-intensity limit). ${ }^{36,37}$ Despite the fact that a stationary soliton requires a $\mathrm{SH}$ component, in almost all of the soliton generation experiments reported to date, only the fundamental is input into the crystal and initially the SH field grows out of phase with the FW. ${ }^{15-20}$ However, because the solitons are the modes of the nonlinear medium at sufficiently high intensities and appropriate beam widths, this combination of fields evolves over distance into a soliton. ${ }^{38}$ The excess harmonic and fundamental energy is radiated away. However, the process is typically slow, so that significant radiation fields are attached to the solitons during several parametric gain and diffraction lengths. ${ }^{45}$ However, any SHG experiment can ultimately lead to soliton generation in the appropriate range of input parameters, primarily the beam width and its intensity. 


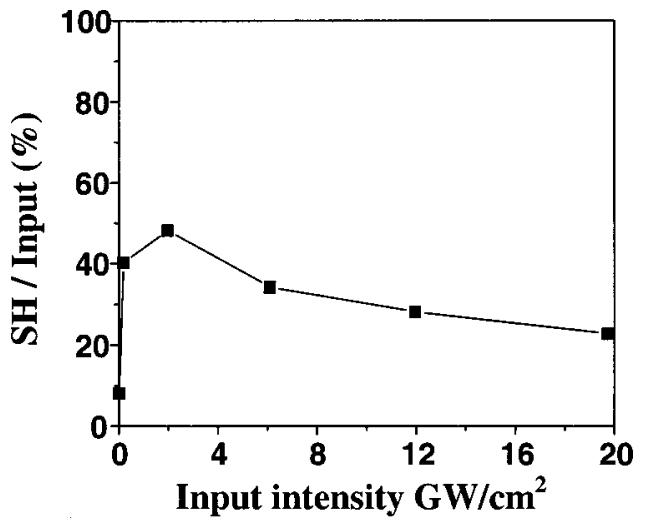

FIG. 6. SHG pulse energy efficiency vs input fundamental intensity at the QPM temperature $\sim 42.7^{\circ} \mathrm{C}$.

\section{TEMPERATURE TUNING MEASUREMENTS}

Numerical modeling of the coupled mode equations was used to qualitatively study the variation in the tuning curves with input beam width and input power for a qualitative comparison with the experiment. Notice that a precise quantitative comparison requires full spatiotemporal simulations, not performed here, to take into account for the pulsed pump conditions. At the negligible depletion regime (i.e., in the conditions of this paper, in the $\mathrm{MW} / \mathrm{cm}^{2}$ range), a factor that averages the pulsed pump conditions can be introduced. However, in the soliton regime pulsed beams can undergo complex spatiotemporal dynamics, making such averaging incorrect. A comprehensive quantitative spatiotemporal study fall beyond the scope of this article, where we focus on the experimental observations, while continuous wave simulations are intended to capture the main trends. Typical situations are shown in Fig. 1(a) for $T<T_{\mathrm{PM}}$, in Fig. 1(b) for $T$ $=T_{\mathrm{PM}}$, and in Fig. 1(c) for $T>T_{\mathrm{PM}}$. The detuning results for $\mathrm{cw}$ excitation are shown in Fig. 3. The calculated tuning curve evolves with decreasing beam width from the classic $\operatorname{sinc}^{2}$ response for plane waves to an asymmetric one with stronger SHG on the side corresponding to $\Delta k>0$. The sig- nificant wave-vector spread associated with the narrower beam leads to the asymmetry observed, as discussed in the introduction and reported previously in the literature. ${ }^{12-14}$ Experimentally, the phase-matching temperature was determined first by measuring the SHG response for a low-input intensity and a relatively wide fundamental beam $\left(w_{0}\right.$ $=40 \mu \mathrm{m}, 1.16$ diffraction lengths of propagation in the crystal). This result, along with that for a $16.4 \mu \mathrm{m}$ beam is shown in Fig. 4(a). Despite the fact that pulsed lasers were used, the $\mathrm{cw}$ trends predicted numerically were reproduced in the experiment.

Numerical results for the cw tuning curves were also obtained for increasing input intensity, Figs. 5(a)-5(c). The response curves broaden with increasing intensity, taking on multipeak structures with no apparent residual of symmetry left. In Fig. 5(d), time averaging over a temporal pulse is also implemented to investigate the deep minimum due to downconversion found in the cw case right on phase match in Fig. 5(b). In selected full spatiotemporal simulations that were performed, the corresponding temporal averaging caused the minimum to no longer exist and in general one would not expect sharp maxima or minima to occur. In good agreement with theory, all semblance of the well-known tuning response disappears when the input intensity is raised to the 1 to $20 \mathrm{GW} / \mathrm{cm}^{2}$ range, as shown in Fig. 4(b). The SHG peak shifts to lower temperatures (to approximately $41{ }^{\circ} \mathrm{C}$ ), a wellknown feature for this case. ${ }^{7,8}$ Furthermore, the response curves broaden and the multiple peak structure becomes progressively more pronounced.

The interpretation of this high-intensity SHG response requires examining many factors including the output beam structure, and the net conversion to $\mathrm{SH}$. Consider first the case of phase matching $\left(42.7^{\circ} \mathrm{C}\right.$ at low-input intensity). The conversion efficiency to second harmonic and the beam profiles for increasing intensity are shown in Figs. 6 and 7, respectively. The fractional conversion of the input energy into second harmonic peaks at $48 \%$ around $2 \mathrm{GW} / \mathrm{cm}^{2}$ of fundamental input intensity, and then decreases with further increase in input intensity to $1 / 2$ of the peak value at 20

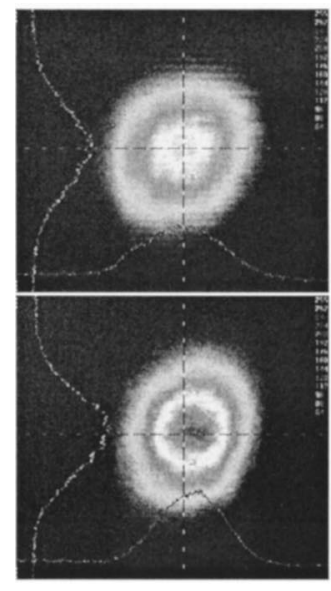

$0.2 \mathrm{GW} / \mathrm{cm}^{2}$

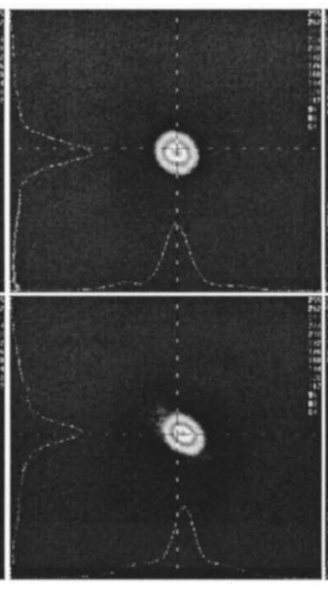

$2 \mathrm{GW} / \mathrm{cm}^{2}$

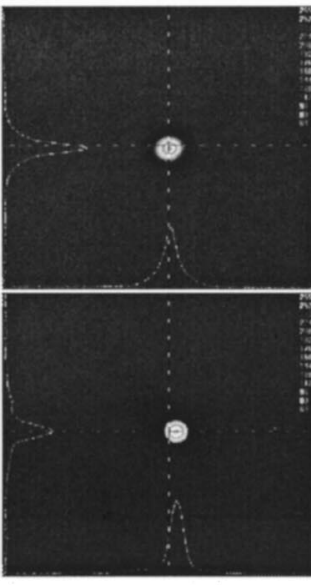

$6.1 \mathrm{GW} / \mathrm{cm}^{2}$

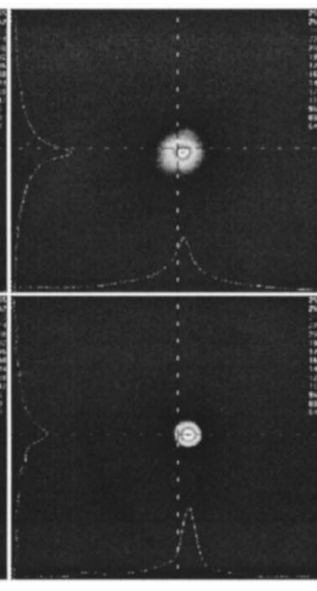

$12 \mathrm{GW} / \mathrm{cm}^{2}$

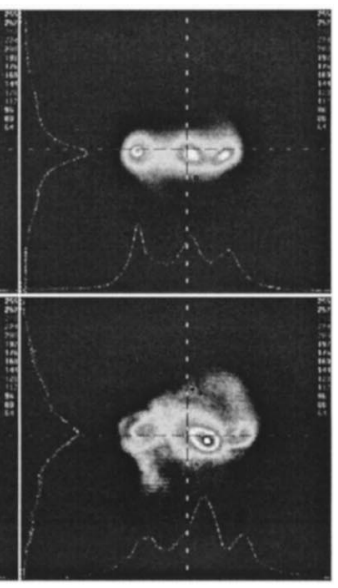

$19.7 \mathrm{GW} / \mathrm{cm}^{2}$

FIG. 7. Fundamental (upper) and second harmonic (lower) output beam profiles for different input intensities on phase-match 


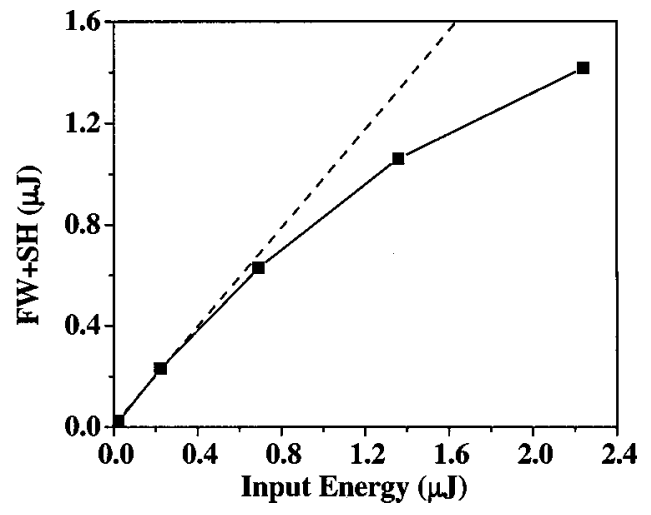

FIG. 8. Total throughput pulse energy for $\mathrm{FW}+\mathrm{SH}$ vs input FW energy at phase-match $\left(42.7^{\circ} \mathrm{C}\right)$.

$\mathrm{GW} / \mathrm{cm}^{2}$, as shown in Fig. 6. From Fig. 7, it is clear that significant narrowing of the fundamental and harmonic begins to occur around a $\mathrm{GW} / \mathrm{cm}^{2}$, essentially coincident with the maximum SHG efficiency. Note also that distortions away from cylindrical symmetry of the SH beam occur despite our best efforts to input cylindrically symmetric beams-for example, the very low-input intensity profiles appear quite symmetric. The threshold for soliton generation is a quantity difficult to measure, as it depends on a number of parameters nonreadily accessible (e.g., because of the pulsed pump conditions, spatial trapping might occur at the peak of the pulse, while diffraction occurs at the wings; as a result, the energy measurements are for an overall broad spatial beam, and thus do not reveal the actual formation of a spatial soliton at the pulse peak $^{46}$ ). An overestimated measure (see Ref. 47) of the threshold is given by the input peak power at which the output beam width coincides with the input value. In our samples and input light conditions (e.g., we used input beams with Gaussian beam quality factors $\mathrm{M}^{2}$ in the range 1.1-1.5), such threshold occurs in the range $\approx 3.0-5.0 \mathrm{GW} / \mathrm{cm}^{2}$ and, as shown in Fig. 7, the soliton dominates the output profile for further increase in intensity. Above $13-15 \mathrm{GW} / \mathrm{cm}^{2}$ several solitons appear at the output.
Finally we note from Fig. 8 that there are significant (20\%) nonlinear losses occurring for intensities above input intensities of $10 \mathrm{GW} / \mathrm{cm}^{2}$, presumably due to two-photon absorption of the $532 \mathrm{~nm}$ component of the soliton. A similar effect has been reported previously in PPKTP at similar input intensities. ${ }^{12}$

Two-photon absorption is not the only important SHG limiting mechanism. Clearly it is the narrowing of the beam into a soliton $\left(1-5 \mathrm{GW} / \mathrm{cm}^{2}\right)$ that also limits the conversion from the fundamental into the harmonic. Note that this narrowing is accompanied by a phase change between the two fields so that when solitons are finally generated the two fields are approximately in phase instead of $\pi / 2$ out of phase, which is the case for the usual SHG on phase match (notice that in the case periodically poled materials, there is a QPMinduced, overall additional $\pi / 2$ phase shift, ${ }^{11}$ not included in this analysis). It is easy to show that in phase fields do not exchange energy efficiently. From the coupled mode equations, replacing $\partial \mathrm{a} / \partial \mathrm{z}$ by $\Delta a / \Delta z$ on phase match gives $\Delta a_{2}$ $\propto-i a_{1}^{2}$ and $\Delta a_{1} \propto-i a_{2} a_{1}$. The factor " $i$ " means that the $\Delta a_{1}$ and $\Delta a_{2}$ are always orthogonal (i.e., $\pi / 2$ out of phase) to $a_{1}$ and $a_{2}$ which leads to a nonlinear rotation of the phase of the two fields and not to net energy exchange between them. ${ }^{48}$ Therefore, the SHG efficiency is limited by the ratio of the harmonic to the fundamental in a soliton since the field amplitudes no longer change with propagation distance due to the stationary nature of solitons, i.e., the fraction of SH in the soliton is fixed. The ratio of SH to FW increases sublinearly with increasing soliton peak intensity. Furthermore the soliton generation process does not necessarily become more efficient with increasing fundamental intensity. The combination of these two effects impacts the fraction of energy converted into SH and affects the shape of the tuning curves. Shown in Figs. 9 and 10 are a collection of FW and SH output beam profiles at different intensities for two temperatures displaces to opposite sides of the phase-matching temperature. Here, "cascading" also plays a role. On the positive wave-vector mismatch side $\left(T<T_{\mathrm{PM}}\right)$, and soliton generation is obtained for an input intensity similar to that on phase

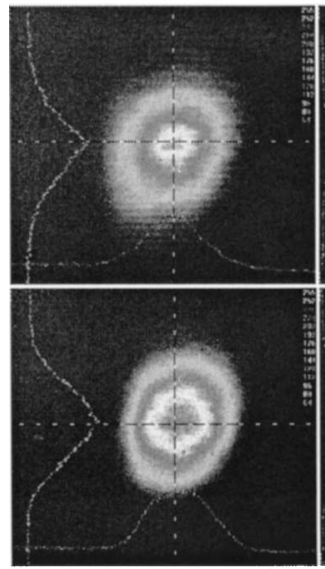

$0.2 \mathrm{GW} / \mathrm{cm}^{2}$

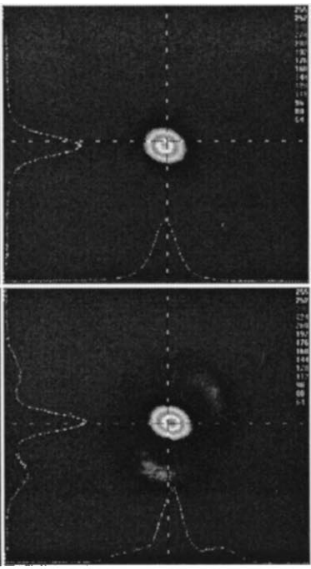

$2 \mathrm{GW} / \mathrm{cm}^{2}$

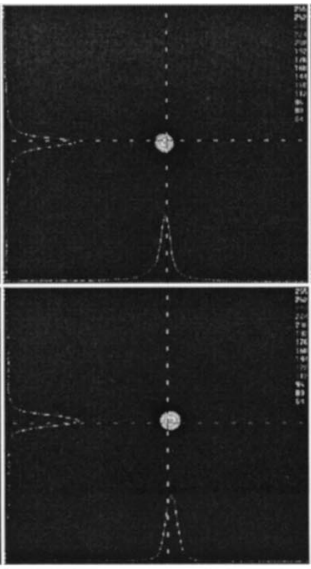

$6.1 \mathrm{GW} / \mathrm{cm}^{2}$

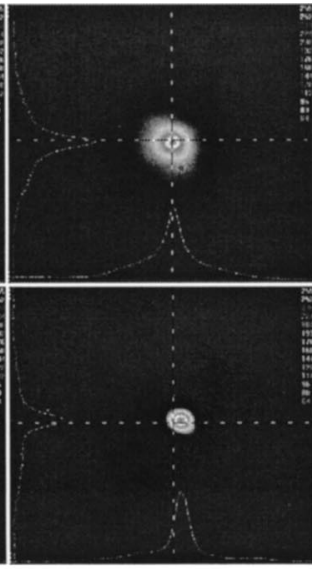

$12 \mathrm{GW} / \mathrm{cm}^{2}$

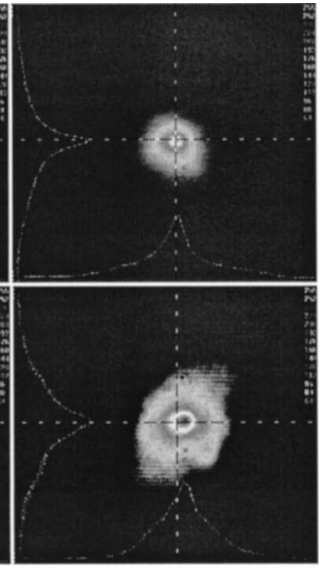

$19.7 \mathrm{GW} / \mathrm{cm}^{2}$

FIG. 9. Fundamental (upper) and second harmonic (lower) output beam profiles for different input intensities at $36.7^{\circ} \mathrm{C}, T<T_{\mathrm{PM}}$. 


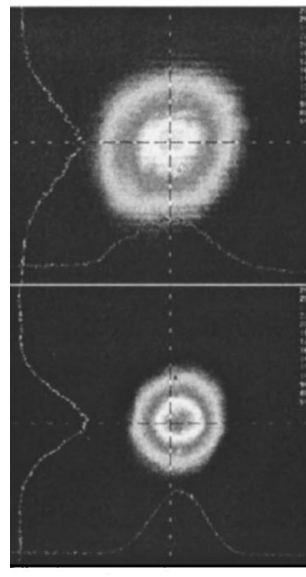

$0.2 \mathrm{GW} / \mathrm{cm}^{2}$

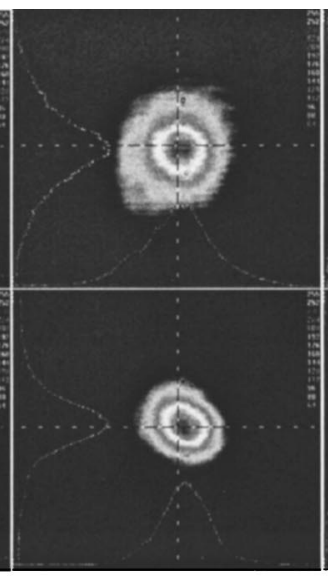

$2 \mathrm{GW} / \mathrm{cm}^{2}$

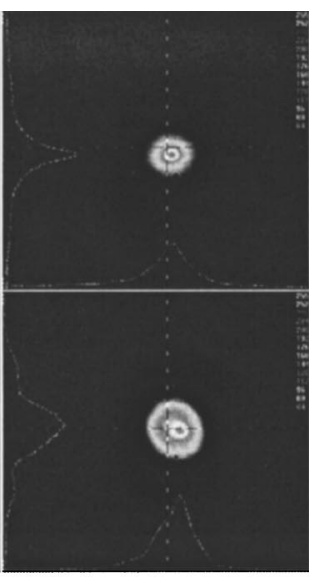

$6.1 \mathrm{GW} / \mathrm{cm}^{2}$

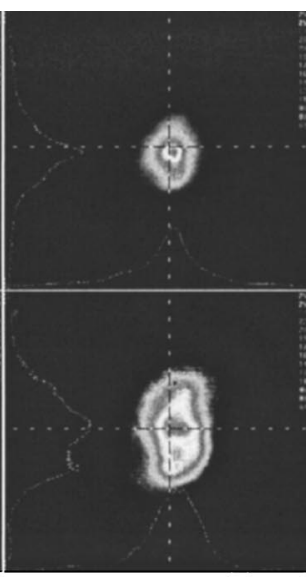

$12 \mathrm{GW} / \mathrm{cm}^{2}$

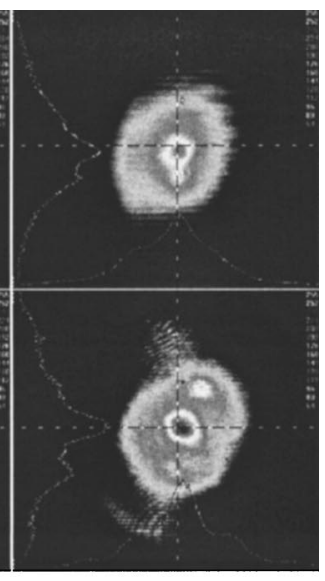

$19.7 \mathrm{GW} / \mathrm{cm}^{2}$

FIG. 10. Fundamental (upper) and second harmonic (lower) output beam profiles for different input intensities at $47.7^{\circ} \mathrm{C}, T>T_{\mathrm{PM}}$.

match. For negative wave-vector mismatch, however, a soliton is much difficult to obtain. These results are in qualitative agreement with previous measurements on soliton threshold intensity with fundamental beam inputs only. ${ }^{15,41-43}$ This is in agreement with the well-known increase in soliton threshold for negative $\Delta k$, and the similar behavior for the ideal soliton content of an incident fundamental beam, shown in Fig. 11 which is skewed to the $\Delta k>0$ side.

Finally we thus conclude that the details of the tuning curve are a consequence of the complex interplay between many phenomena such as the beam narrowing, cascading, soliton generation, relative phase between the fields (in phase for solitons), etc. Further complications arise in the presence of temperature, hence wave vector gradients along the sample. $^{24}$

\section{ANGLE TUNING}

The two principal cases of interest for angle tuning, i.e., for $T=T_{\mathrm{PM}}$ and $T<T_{\mathrm{PM}}$ were investigated numerically at

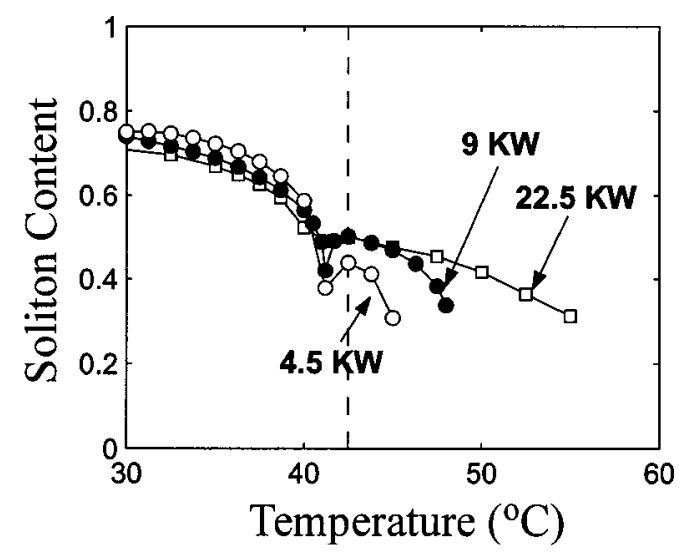

FIG. 11. Calculated soliton content (namely, the fraction of power carried by the soliton divided by the total input power) for fundamental only excitation vs temperature, for ideal conditions of continuous-wave pump light with a spatial Gaussian shape, no losses, and infinite sample lengths. The dashed line corresponds to exact phase-matching. Open circles: 0.9 $\mathrm{GW} / \mathrm{cm}^{2}$; filled circles: $1.75 \mathrm{GW} / \mathrm{cm}^{2}$; and squares: $4.4 \mathrm{GW} / \mathrm{cm}^{2}$. high intensities for cw inputs. The results, which as above are only intended to show qualitative trends, are shown in Fig. 12. For $T=T_{\mathrm{PM}}$ this corresponds to the situation in Fig. 1(b). Since the $z$ axis is a symmetry axis, one expects the tuning curves to be symmetric about the $x$ axis. Off axis the tuning corresponds to $\Delta k<0$. Note that there is walk-off associated with this geometry due to the QPM wave vector not being collinear with those of the fundamental or harmonic. ${ }^{11}$ The SHG tuning response is strongly broadened at the phase-matching temperature due to the high intensity assumed, as discussed above.
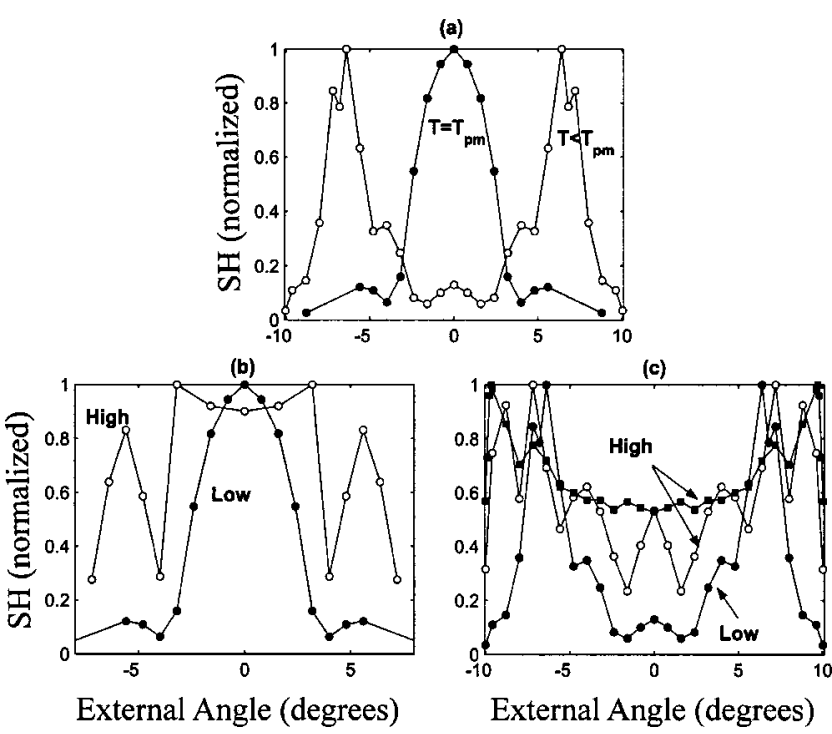

FIG. 12. Calculated angle tuning SHG response for continuous-wave excitation for $T=T_{\mathrm{PM}}, T<T_{\mathrm{PM}}$ and different input intensities. The beam width was fixed to $18 \mu \mathrm{m}$, and the crystal length to $10 \mathrm{~mm}$. In (a), low-input power of $0.46 \mathrm{~W}\left(90 \mathrm{KW} / \mathrm{cm}^{2}\right)$; filled circles, $T=T_{\mathrm{PM}}$ and open circles, $T$ $<T_{\mathrm{PM}}$. In (b), $T=T_{\mathrm{PM}}$; filled circles: low input powers of $0.46 \mathrm{~W}$ (90 $\left.\mathrm{KW} / \mathrm{cm}^{2}\right)$; open circles: high powers of $1.14 \mathrm{KW}\left(225 \mathrm{MW} / \mathrm{cm}^{2}\right)$. In (c), $T<T_{\mathrm{PM}}$; filled circles: low-input powers of $0.46 \mathrm{~W}\left(90 \mathrm{KW} / \mathrm{cm}^{2}\right)$; open circles: high powers of $5.5 \mathrm{KW}\left(1 \mathrm{GW} / \mathrm{cm}^{2}\right)$; filled squares: high powers of $18.3 \mathrm{KW}\left(3.6 \mathrm{GW} / \mathrm{cm}^{2}\right)$. 


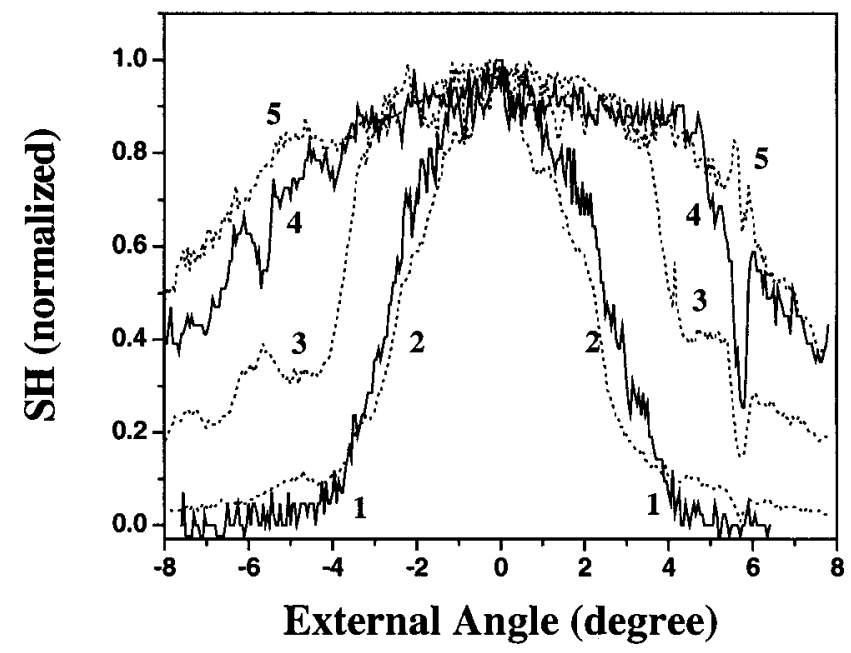

FIG. 13. SHG tuning curve vs incidence angle in the X-Y plane at $42.7^{\circ} \mathrm{C}$ $\left(T=T_{\mathrm{pm}}\right) .0^{\circ}$ angle corresponds to phase-match. 1-0.02, 2-0.2, 3-2.0, $4-6.1$, and $5-19.7 \mathrm{GW} / \mathrm{cm}^{2}$.

Experimentally we investigated the SHG tuning curves when the incidence angle is tuned in the X-Y plane of the crystal at the phase-matching temperature. The most salient feature observed in Fig. 13 is the intensity-dependent broadening associated with strong nonlinear beam focusing effects for sample lengths longer than the parametric gain length. This is in agreement with the trends predicted in Fig. 12. Note that the largest changes occur near the soliton threshold where the beam collapse is a dominant effect, and that at higher intensities the response stabilizes. A similar stabilization was found in the temperature tuning results subsequent to soliton formation.

At room temperature, the situation for index matching is shown in Fig. 1(a). For $T<T_{\mathrm{PM}}$, corresponding to Fig. 1(a), two phase-matching peaks, symmetrically displaced around the $x$ axis, are predicted with almost a constant SHG efficiency between them. Experimentally, decreasing the width of the input beam leads to asymmetry about a phasematching peak, as shown in Fig. 14. The angle region between the two peaks corresponds to $\Delta k>0$, and outside the peaks to $\Delta k<0$. In keeping again with previous measure-

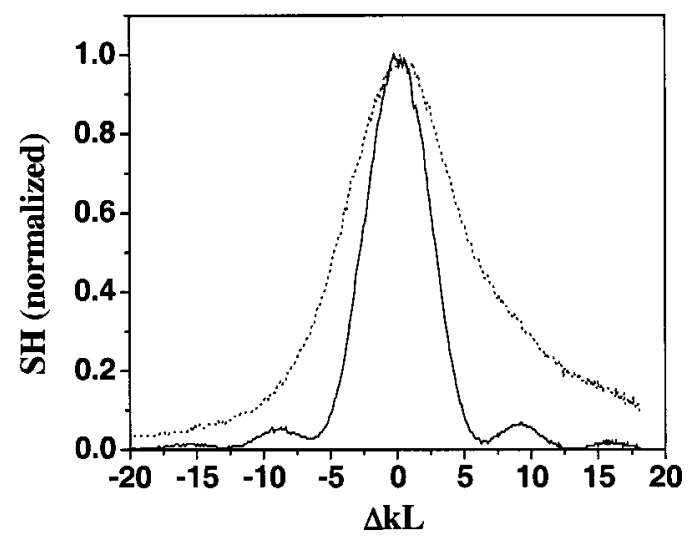

FIG. 14. Angle tuning near the phase-match condition $\left(\theta=8.8^{\circ}\right)$ at room temperature. The solid line is for a $\mathrm{w}_{0}=40 \mu \mathrm{m}$ and the dotted line for $\mathrm{w}_{0}$ $=16 \mu \mathrm{m}$.

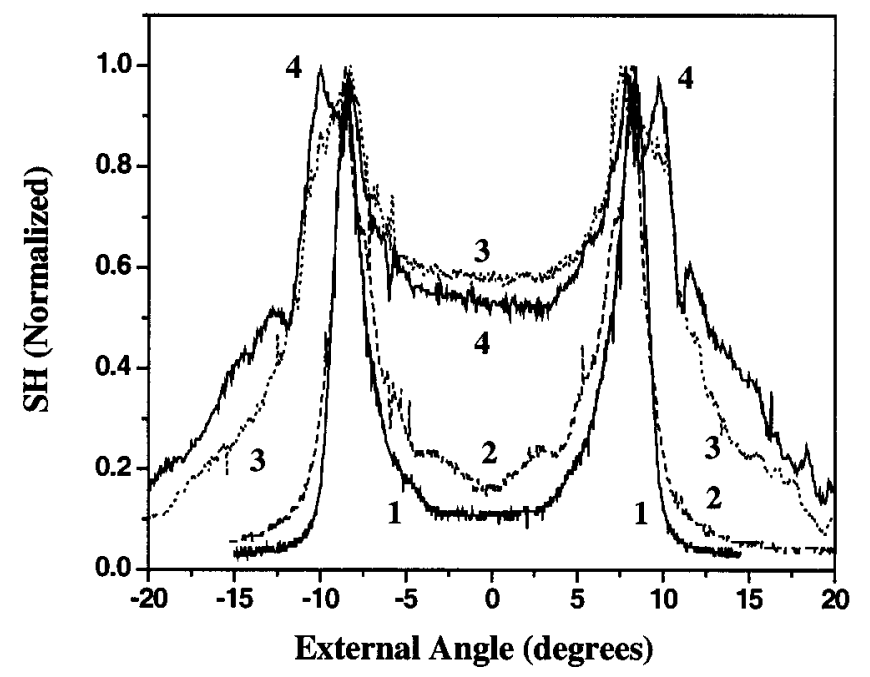

FIG. 15. SHG detuning curves at room temperature for different fundamental input intensities. $1-0.02,2-0.9,3-6.45$, and $4-12.9 \mathrm{GW} / \mathrm{cm}^{2}$.

ments of soliton thresholds and the predictions of soliton content for fundamental wave excitation, solitons were easily excited for $\Delta k>0$. For $\Delta k<0$, soliton excitation required progressively more input intensity.

The intensity dependence of the tuning curves is shown in Fig. 15. Both a clear broadening with increasing intensity and a stabilization of the curves for intensities above the soliton threshold are observed. Here all three effects known to affect the beam profiles are active, i.e., beam narrowing, cascading, and walk-off. The evolution of the output beam profile at a number of representative input intensities is shown in Fig. 16. Here the QPM-induced walk-off angle in the $\mathrm{X}-\mathrm{Y}$ plane is 0.27 degrees, external angle. It is wellknown from previous work in regimes with walk-off that at sufficiently high intensities the two beams "lock" together to form a soliton and propagate in the same direction in space. ${ }^{15,49,50}$ The locking is clear at intensities above $\approx 5$ $\mathrm{GW} / \mathrm{cm}^{2}$ where the two beams propagate together and their direction appears to become stable based on measurements shown in Fig. 16. (The slight artificial displacement between the FW and the SH in the pictures is an experimental artifact.) Especially interesting is the second peak which appears at $\approx 9.9^{\circ}$, at higher angles than the original phase-matching peak $\left(8.84^{\circ}\right)$. The two peaks have an equal SHG efficiency over a limited range of input intensities. Such a sharp minimum between the peaks was not expected due to the smoothing effects caused by time averaging of the temporal pulse profiles. This angular region was explored at an input intensity of $\approx 10 \mathrm{GW} / \mathrm{cm}^{2}$ at which the second peak just occurs. Shown in Fig. 17 are output beam profiles as the angle is tuned through these two peaks. At the minimum between the two peaks, both the FW and SH energy are split into two fragments, one of which appears to be a distorted soliton. At the two peaks, the beams are both still distorted although the energy is more localized than in the minimum.

\section{SUMMARY}

We have experimentally investigated the SHG tuning curves in PPKTP at input intensities comparable to those 


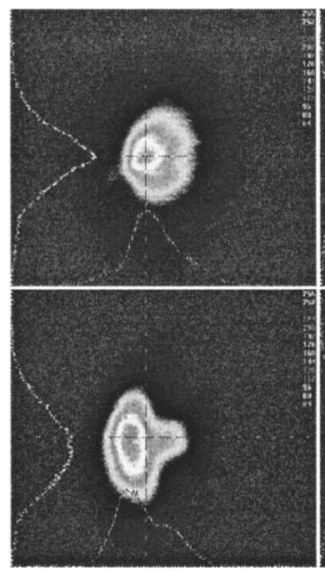

$0.95 \mathrm{GW} / \mathrm{cm}^{2}$

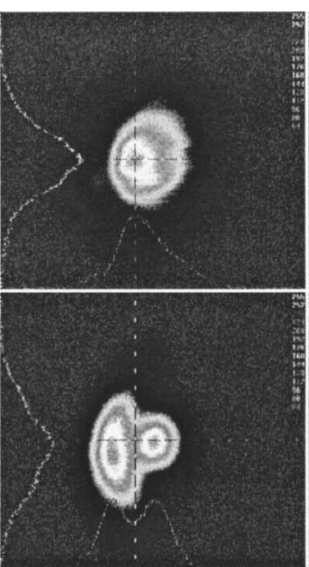

$1.11 \mathrm{GW} / \mathrm{cm}^{2}$

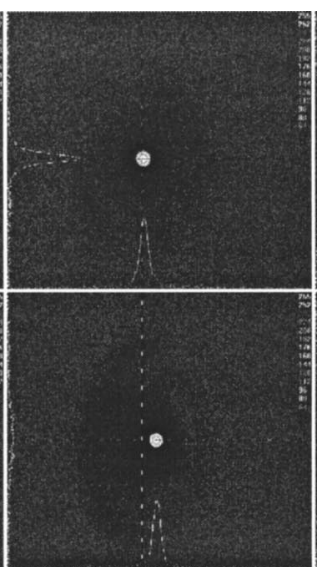

$6.67 \mathrm{GW} / \mathrm{cm}^{2}$

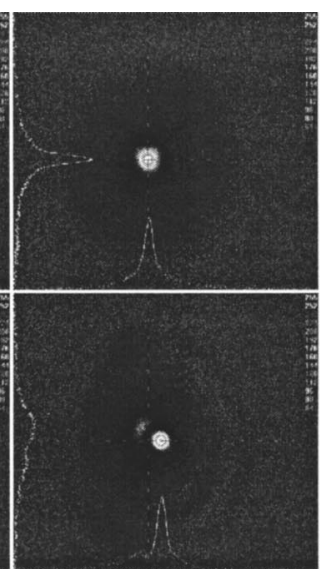

$12.7 \mathrm{GW} / \mathrm{cm}^{2}$

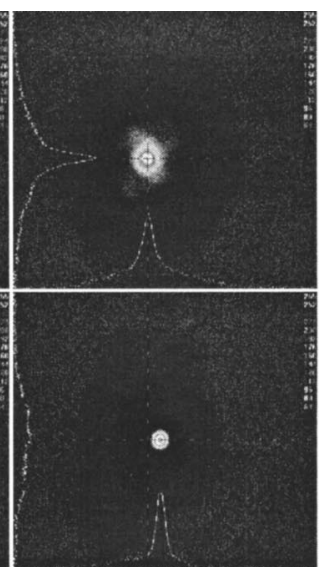

$18.4 \mathrm{GW} / \mathrm{cm}^{2}$

FIG. 16. Various output beam profiles, upper FW and lower SH, on the phase-matching peak $\left(8.84^{\circ}\right)$ for angle tuning at room temperature.

needed for quadratic soliton excitation. The mutual beam narrowing of the fundamental and second harmonic was found to strongly impact the SHG efficiency. The SHG efficiency peaks at intensities for which beam narrowing is large and at higher intensities the SHG decreases with input intensity due primarily to the formation of solitons from fundamental only inputs. The situation becomes more complex for temperature tuning, but with propagation still along the QPM axis. The cascading effect, together with the wave-vector dependence of the soliton threshold, lead to additional asymmetries in the tuning curves. The most striking effect, however, is an intensity-dependent broadening of the tuning curves and the appearance of strong modulation of the SHG, especially on the negative wave-vector mismatch side where it is accompanied by large beam distortion. Angle tuning with $\Delta k>0$ on the $\mathrm{X}$ axis was further complicated by the presence of QPM-induced walk-off between the fundamental and harmonic. The beam distortion now occurred primarily in the plane in which walk-off occurred. Such walk-off was

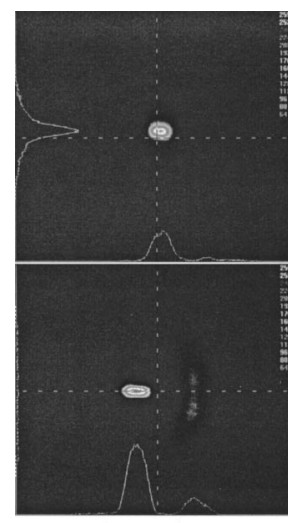

$8.84^{\circ}$

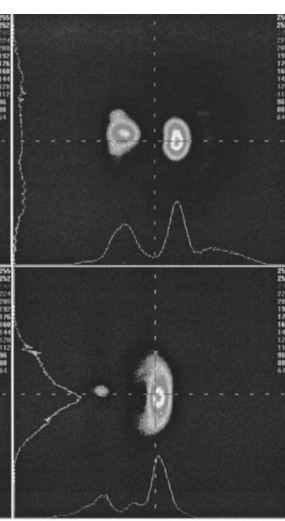

$9.18^{\circ}$

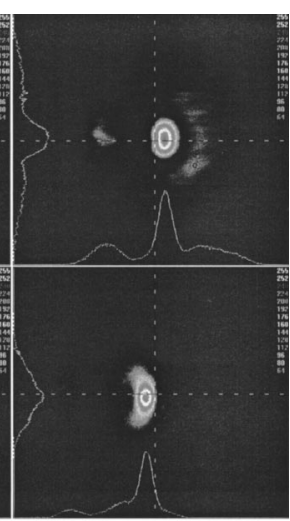

$9.36^{\circ}$
FIG. 17. Various output beam profiles, upper FW and lower $\mathrm{SH}$, on the original phase-matching peak $\left(8.84^{\circ}\right)$, the secondary peak $\left(9.36^{\circ}\right)$, and in the minimum between the two peaks $\left(9.18^{\circ}\right)$ for angle tuning at room temperature. The fundamental input intensity is $9.7 \mathrm{GW} / \mathrm{cm}^{2}$. found to lead to distortion of the soliton profiles even at input intensities higher that that associated with the soliton threshold.

\section{ACKNOWLEDGMENTS}

The experimental research in the United States was supported by an Army Research Office MURI, and the collaboration between the American and Spanish investigators was made possible by the Commission for Scientific Exchange between the United States and Spain. H.K. was supported by a postdoctoral fellowship program from Korea Science \& Engineering Foundation (KOSEF). S.C. and L.T. were supported by the Generalitat de Catalunya and by the Spanish Government under Contract No. TIC2000-1010.

${ }^{1}$ C. L. Tang, W. R. Bosenberg, T. Ukachi, R. J. Lane, and L. K. Cheng, Proc. IEEE 80, 365 (1992).

${ }^{2}$ F. A. Hopf and G. I. Stegeman, Applied Classical Electrodynamics, Vol. 2: Nonlinear Optics (Wiley, New York, 1986), pp. 1-74.

${ }^{3}$ Y. R. Shen, The Principles of Nonlinear Optics (Wiley, New York, 1984), pp. 67-140.

${ }^{4}$ For example, R. C. Eckard and J. Reintjes, IEEE J. Quantum Electron. 20, 1178 (1984)

${ }^{5}$ A recent modern example is K. R. Parameswaran, J. R. Kurz, R. V. Roussev, and M. M. Fejer, Opt. Lett. 27, 43 (2002).

${ }^{6}$ K. R. Parameswaran, J. R. Kurz, R. V. Roussev, and M. M. Fejer, Digest of Topical Meeting on 2002 Nonlinear Optics (Opt. Soc. Am., Washington, 2002), paper TuB6.

${ }^{7}$ D. A. Kleinman, A. Ashkin, and G. D. Boyd, Phys. Rev. 145, 338 (1966).

${ }^{8}$ G. D. Boyd and D. A. Kleinman, J. Appl. Phys. 39, 3597 (1968).

${ }^{9}$ S. Guha, F.-J. Wu, and J. Falk, IEEE J. Quantum Electron. QE-18, 907 (1982).

${ }^{10}$ T. Suhara and H. Nishihara, IEEE J. Quantum Electron. 26, 1265 (1990).

${ }^{11}$ M. M. Fejer, G. A. Magel, D. H. Jundt, and R. L. Byer, IEEE J. Quantum Electron. 28, 2631 (1992)

${ }^{12}$ Y. Wang, V. Petrov, Y. J. Ding, Y. Zheng, J. B. Khurgin, and W. P. Risk, Appl. Phys. Lett. 73, 873 (1998).

${ }^{13}$ G. M. Gibson, G. A. Turnbull, M. Ebrahimzadeh, M. H. Dunn, H. Karlsson, G. Arvidsson, and F. Laurell, Appl. Phys. B: Lasers Opt. 67, 675 (1998).

${ }^{14}$ K. Fradkin, A. Arie, A. Skliar, and G. Rosenman, Appl. Phys. Lett. 74, 914 (1999).

${ }^{15}$ W. E. Torruellas, Z. Wang, D. J. Hagan, E. W. VanStryland, G. I. Stegeman, L. Torner, and C. R. Menyuk, Phys. Rev. Lett. 74, 5036 (1995).

${ }^{16}$ R. Schiek, Y. Baek, and G. I. Stegeman, Phys. Rev. E 53, 1138 (1996). 
${ }^{17}$ P. Di Trapani, G. Valiulis, W. Chianglia, and A. Adreoni, Phys. Rev. Lett. 80, 265 (1998).

${ }^{18}$ X. Liu, L. J. Qian, and F. W. Wise, Phys. Rev. Lett. 82, 4631 (1999).

${ }^{19}$ B. Bourliaguet, V. Couderc, A. Barthelemy, G. W. Ross, P. G. R. Smith, D.

C. Hanna, and C. De Angelis, Opt. Lett. 24, 1410 (1999).

${ }^{20}$ R. Malendevich, L. Jankovic, S. Polyakov, R. Fuerst, G. I. Stegeman, Ch. Bosshard, and P. Gunter, Opt. Lett. 27, 631 (2002).

${ }^{21}$ Yu. N. Karamzin and A. P. Sukhorukov, Zh. Eksp. Teor. Phys 68, 834-40 (1975) [Sov. Phys. JETP 41, 414 (1976)].

${ }^{22}$ Reviewed in G. I. Stegeman, D. J. Hagan, and L. Torner, Opt. Quantum Electron. 28, 1691 (1996).

${ }^{23}$ L. A. Ostrovskii, Zh. Eksp. Teor. Fiz., Pis'ma Red. 5, 9 (1967) [JETP Lett. 5, 272 (1967)].

${ }^{24}$ R. Schiek, Y. Baek, and G. I. Stegeman, J. Opt. Soc. Am. B 15, 2255 (1998).

${ }^{25}$ G. Rosenman, Kh. Garb, A. Skliar, M. Oron, D. Eger, and M. Katz, Appl. Phys. Lett. 73, 865 (1998).

${ }^{26}$ G. Rosenman, A. Skliar, D. Eger, M. Oron, and M. Katz, Appl. Phys. Lett. 73, 3650 (1998)

${ }^{27}$ H. Vanherzeele and J. D. Bierlein, Opt. Lett. 17, 982 (1992).

${ }^{28}$ A. Arie, G. Rosenman, V. Mahal, A. Skliar, M. Oron, M. Katz, and D. Eger, Opt. Commun. 142, 265 (1997).

${ }^{29}$ A. Englander, R. Lavi, M. Katz, M. Oron, D. Eger, E. Lebiush, G. Rosenman, and A. Skliar, Opt. Lett. 22, 1598 (1997).

${ }^{30}$ A. Arie, G. Rosenman, A. Korenfield, A. Skliar, M. Oron, M. Katz, and D. Eger, Opt. Lett. 23, 28 (1998).

${ }^{31}$ M. Katz, D. Eger, M. B. Oron, and A. Hardy, J. Appl. Phys. 90, 53 (2001).

${ }^{32}$ W. Wiechmann, S. Kubota, T. Fukui, and H. Masuda, Opt. Lett. 18, 1208 (1993).

${ }^{33}$ J. A. Armstrong, N. Bloembergen, J. Ducuing, and P. S. Pershan, Phys. Rev. 127, 1918 (1962).
${ }^{34}$ C. R. Menyuk, R. Schiek, and L. Torner, J. Opt. Soc. Am. B 11, 2434 (1994).

${ }^{35}$ G. I. Stegeman, J. Opt. A, Pure Appl. Opt. 9, 139 (1997).

${ }^{36}$ G. I. Stegeman, M. Sheik-Bahae, E. VanStryland, and G. Assanto, Opt. Lett. 18, 13 (1993).

${ }^{37}$ A. V. Buryak and Y. S. Kivshar, Opt. Lett. 19, 1612 (1994).

${ }^{38}$ L. Torner, C. R. Menyuk, and G. I. Stegeman, Opt. Lett. 19, 1615 (1994).

${ }^{39}$ L. Torner, Opt. Commun. 114, 136 (1995).

${ }^{40}$ A. V. Buryak and Y. S. Kivshar, Phys. Lett. A 197, 407 (1995).

${ }^{41}$ R. A. Fuerst, M. T. G. Canva, D. Baboiu, and G. I. Stegeman, Opt. Lett. 22, 1748 (1997).

${ }^{42}$ R. A. Fuerst, M. T. G. Canva, G. I. Stegeman, G. Leo, and G. Assanto, Opt. Quantum Electron. 30, 907 (1998).

${ }^{43}$ P. Di Trapani, A. Bramati, S. Minardi, W. Chinaglia, C. Conti, S. Trillo, J. Kilius, and G. Valiulis, Phys. Rev. Lett. 87, 183902 (2001).

${ }^{44}$ W. Torruellas, Y. Kivshar, and G. I. Stegeman, Spatial Solitons, edited by S. Trillo and W. Torruellas (Springer-Verlag, Berlin, 2001), Chap. 6, p. 127.

${ }^{45}$ L. Torner, J. P. Torres, D. Artigas, D. Mihalache, and D. Mazilu, Opt. Commun. 164, 153 (1999).

${ }^{46}$ S. Carrasco, J. P. Torres, D. Artigas, and L. Torner, Opt. Commun. 192, 347 (2001).

${ }^{47}$ E. Lopez-Lago, C. Simos, V. Couderc, A. Barthelemy, D. Artigas, and L. Torner, Opt. Lett. 26, 1277 (2001).

${ }^{48}$ G. Assanto and G. I. Stegeman, Opt. Express 10, 388 (2002).

${ }^{49}$ L. Torner, D. Mazilu, and D. Mihalache, Phys. Rev. Lett. 77, 2455 (1996).

${ }^{50}$ R. Schiek, Y. Baek, G. I. Stegeman, I. Baumann, and W. Sohler, Opt. Lett. 24, 83 (1999). 